\title{
Two-Dimensional Optical Control of Electron Spin Orientation by Linearly Polarized Light in InGaAs
}

\author{
K. Schmalbuch, ${ }^{1,2}$ S. Göbbels, ${ }^{1,2}$ Ph. Schäfers, ${ }^{1,2}$ Ch. Rodenbücher, ${ }^{1,2}$ P. Schlammes, ${ }^{1,2}$ \\ Th. Schäpers, ${ }^{3,2}$ M. Lepsa, ${ }^{3,2}$ G. Güntherodt, ${ }^{1,2}$ and B. Beschoten ${ }^{1,2, *}$ \\ ${ }^{1}$ II. Institute of Physics, RWTH Aachen University, Otto-Blumenthal-Straße, 52074 Aachen, Germany \\ ${ }^{2}$ JARA: Fundamentals of Future Information Technology, Jülich-Aachen Research Alliance, Aachen, Germany \\ ${ }^{3}$ Institute of Bio- and Nanosystems, Forschungszentrum Jülich, 52428 Jülich, Germany
}

(Received 1 August 2010; published 7 December 2010)

\begin{abstract}
Optical absorption of circularly polarized light is well known to yield an electron spin polarization in direct band gap semiconductors. We demonstrate that electron spins can even be generated with high efficiency by absorption of linearly polarized light in $\mathrm{In}_{x} \mathrm{Ga}_{1-x}$ As. By changing the incident linear polarization direction we can selectively excite spins in both polar and transverse directions. These directions can be identified by the phase during spin precession using time-resolved Faraday rotation. We show that the spin orientations do not depend on the crystal axes suggesting an extrinsic excitation mechanism.
\end{abstract}

DOI: 10.1103/PhysRevLett.105.246603

The generation of spin-polarized charge carriers by optical orientation in nonmagnetic semiconductors is well established. In optical orientation the angular momentum of circularly polarized photons will be transferred to electrons and holes during absorption [1,2]. This can result in a large spin polarization of $50 \%$ in bulk III-V semiconductors. Besides static imaging and probing of the spin polarization [3,4], optical pump-probe measurements using time-resolved Faraday rotation (TRFR) have become a standard method for both triggering and probing of spin coherence in semiconductors [5-10]. In contrast, optical absorption of linearly polarized photons should not result in net spin polarization as an equal number of spin-up and spin-down electrons and holes will be generated. It has been predicted, however, that in quantum well structures of reduced symmetry optical spin orientation by linearly polarized light might be feasible due to zero-field spin splitting of electron and hole states [11]. It is furthermore known that linearly polarized light can yield a spindependent photovoltage response originating from spin photogalvanic effects $[12,13]$. However, those spin polarizations have not been probed by magneto-optical methods.

In this Letter we report on measurements of electron spin coherence in InGaAs by TRFR after optical orientation by linearly polarized laser pulses. We observe both polar and transverse initial spin orientations, which can be controlled independently as a function of the incident linear polarization direction. The number of transverse spins increases linearly with the magnitude of a perpendicular external magnetic field and vanishes at zero field, while the number of polar spins is unaffected by the external magnetic field. We demonstrate that the optical orientation by linearly polarized light has a comparable efficiency as that by circularly polarized light. The
PACS numbers: 72.25.Fe, 42.50.Md, 76.30.Pk, 78.47.-p

generated respective spin orientations are furthermore found to be independent of the crystal orientation of the InGaAs layer, indicating an extrinsic excitation mechanism.

We have studied several $\operatorname{In}_{x} \mathrm{Ga}_{1-x}$ As samples with In contents $0 \leq x \leq 0.1$ and thicknesses between $300 \mathrm{~nm}$ and $1 \mu \mathrm{m}$, which are grown by molecular beam epitaxy on semi-insulating (001)GaAs substrates. The room temperature carrier density was set to $n \sim 3 \times 10^{16} \mathrm{~cm}^{-3}$ by Si doping to allow for long spin dephasing times at low temperatures [4,5]. Note that all presented results have been observed in all samples independent of In content and thickness. In the following, we show representative data, which were taken on a $500 \mathrm{~nm}$ epilayer of $\mathrm{In}_{x} \mathrm{Ga}_{1-x} \mathrm{As}$ with $x=4.9 \%$. The sample was mounted strain-free in an optical He-flow cryostat. Phase triggering of electron spin coherence is achieved by either circularly or linearly polarized picosecond pump pulses. The incident polarization direction of the latter can be changed continuously by an angle $\varphi$ as defined in Fig. 1(a). Spin precession is probed in a transverse external magnetic field $B$ (oriented along the $y$ direction) by a second time-delayed linearly polarized probe pulse using standard measurements of the TRFR angle $\theta_{F}$, which is a measure of the polar spin component. Its time dependent evolution can be described by an exponentially damped cosine function

$$
\theta_{F}(\Delta t)=\theta_{0} \exp \left(-\frac{\Delta t}{T_{2}^{*}}\right) \cos \left(\omega_{L} \Delta t+\delta\right)
$$

with amplitude $\theta_{0}$, transverse spin dephasing time $T_{2}^{*}$, Larmor frequency $\omega_{L}=g \mu_{B} B / \hbar$, time delay $\Delta t$ between the pump and probe pulses and phase factor $\delta . g$ is the effective electron $g$ factor, $\mu_{B}$ the Bohr magneton, and $\hbar$ the Planck constant. The sample plane can furthermore be rotated by an angle $\alpha$ about the $x$ axis. 
(a)

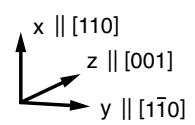

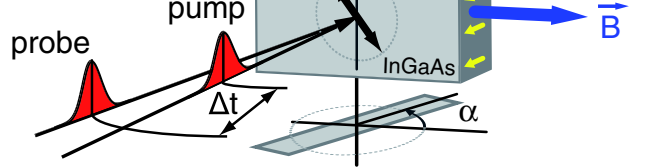

(b)

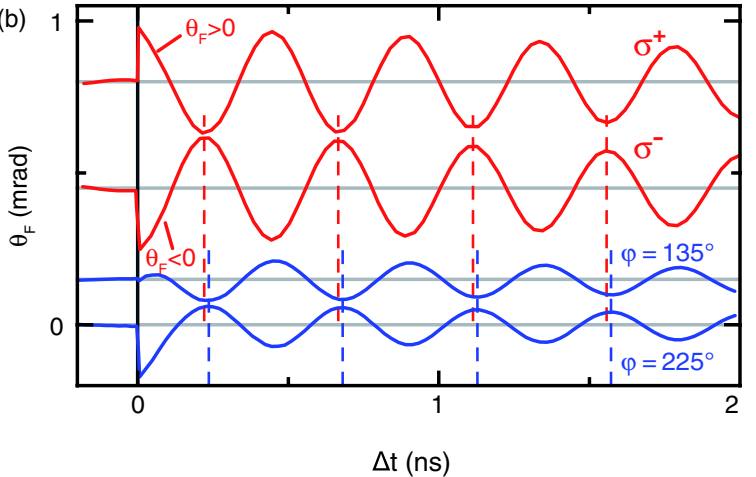

FIG. 1 (color online). (a) Setup for all-optical measurements of TRFR using linearly polarized pump pulses. The incident linear polarization direction can continuously be adjusted by the angle $\varphi$. (b) Comparison of TRFR after excitation by circularly [light gray (red) curves] and linearly [dark gray (blue) curves] polarized pump pulses. Data are taken at $T=30 \mathrm{~K}$ in a transverse magnetic field of $0.5 \mathrm{~T}$. The dashed vertical lines indicate a phase shift in the precession between circular and linear excitation. A vertical offset is added for clarity.

Figure 1(b) depicts TRFR measurements after optical excitation with both circularly and linearly polarized pump pulses using a laser energy near the fundamental band gap of the InGaAs layer. Data were taken at $T=30 \mathrm{~K}$ and $B=0.5 \mathrm{~T}$. When using circularly polarized light the angular momentum of the photon will be absorbed during interband absorption resulting in spin-polarized electrons and holes. The holes will be ignored in the following discussion. By changing the light helicity from $\sigma^{+}$to $\sigma^{-}$, we can control the initial spin orientation between parallel and antiparallel alignment relative to the incident light propagation direction. The resulting TRFR measurements are depicted in Fig. 1(b) [light gray (red) curves] for $\sigma^{+}$and $\sigma^{-}$laser excitation under nearly normal incidence. The change of the initial spin orientation is easily seen by a sign change of $\theta_{F}$ right after excitation $(\Delta t=0 \mathrm{~ns})$. Note that we measure $\theta_{F}$ in the polar geometry for all presented experiments. We are thus only sensitive to spin components, which are pointing in the $\pm z$ direction. When using linearly polarized pump pulses, we would not expect to excite a net spin polarization as the linearly polarized light is a superposition of $\sigma^{+}$and $\sigma^{-}$photons, which results in an equal number of spin-up and spin-down electrons after absorption. In Fig. 1(b) we show TRFR data taken for normal incidence $\alpha=0^{\circ}$ at two distinct polarization angles of linearly polarized pump pulses, which differ by $90^{\circ}$ [dark gray (blue) curves]. We, however, clearly observe spin precession. Surprisingly, the amplitude is only slightly reduced compared to the curves taken under $\sigma^{+}$and $\sigma^{-}$ excitation demonstrating that optical orientation of electron spins by linearly polarized light is strikingly efficient.

We note that spins of opposite directions can be excited when changing the polarization by $90^{\circ}$ from $135^{\circ}$ to $225^{\circ}$. While the precession frequency is identical for all excitations, we observe a phase shift in the precession for linear polarized excitation as indicated by the vertical dashed lines in the $\sigma^{+}$and $\sigma^{-}$curves in Fig. 1(b). Such a phase shift indicates a change of the initial spin direction.

To further explore the polarization dependence of $\theta_{F}$, we plot a series of TRFR measurements with $\varphi$ varying between $0^{\circ}$ and $360^{\circ}$ in Fig. 2(a). The most striking observation is the sign reversal of $\theta_{F}$ every $90^{\circ}$ [see also Fig. 4(a)], which is further investigated in Figs. 2(b) and 2(c), where the polarization angle resolution is enhanced in a regime of sign reversal. It is clearly seen that spin precession is observed at all angles. The sign reversal of $\theta_{F}$ between point $A\left(\varphi=280.2^{\circ}\right.$, red color code $\left.\theta_{F}>0^{\circ}\right)$ and point $B\left(\varphi=275.8^{\circ}\right.$, blue color code $\left.\theta_{F}<0^{\circ}\right)$ in Fig. 2(c) is accompanied by a continuous change of the phase $\delta$ of the precessing spins [see also dotted line in Fig. 2(b)]. The phase can be extracted from fitting all TRFR traces in Fig. 2(c) by Eq. (1). As seen in Fig. 3(a), the phase continuously changes from $-180^{\circ}$ to almost $0^{\circ}$ within a small range of linear polarization angles. We want to emphasize that in our polar configuration we only probe spins, which have a finite projection along the $\pm z$ axis. For $\delta=0^{\circ}$, spins are oriented in the polar $+z$ direction at $\Delta t=0$, which results in a TRFR curve starting in a positive maximum [see also Fig. 3(b)]. On the other hand, spins are oriented in the $-z$ direction for
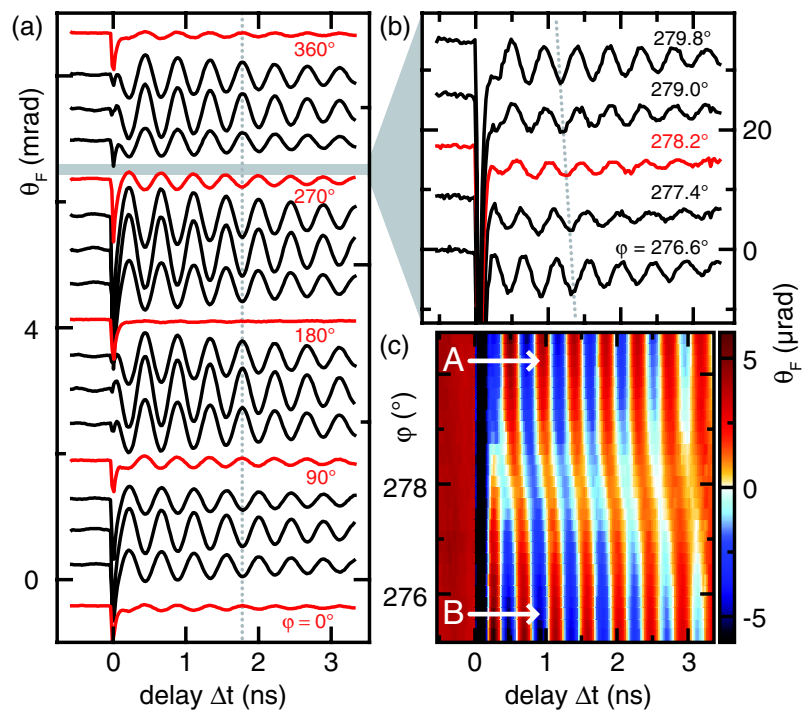

FIG. 2 (color online). TRFR for optical excitation with linearly polarized laser pulses measured in $n$-InGaAs at $T=30 \mathrm{~K}$ and $B=0.5 \mathrm{~T}$ for (a) $\varphi=0^{\circ}-360^{\circ}$ (the vertical dotted line helps to see the sign reversal every $90^{\circ}$ ). The sign reversal is accompanied by a continuous phase shift of the precessing spins, which can be seen in (b) and (c). 


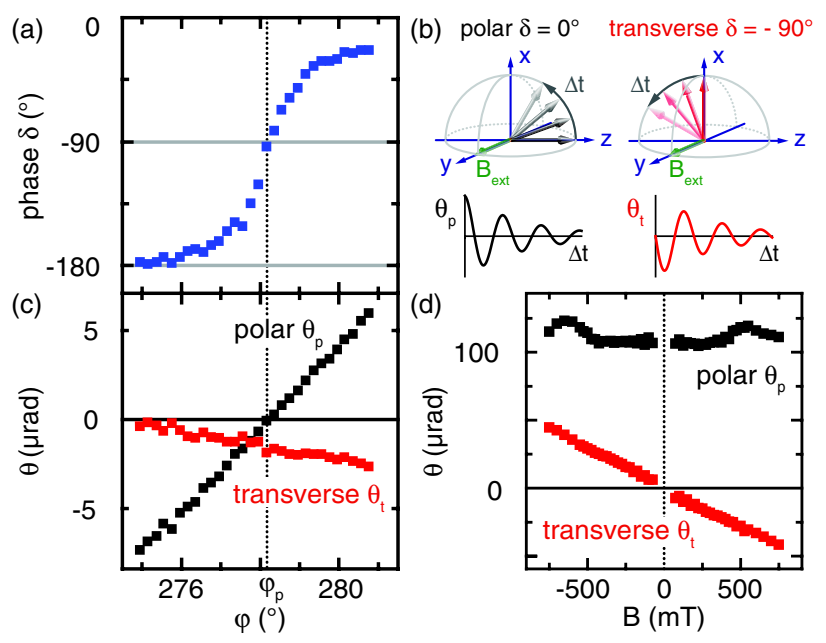

FIG. 3 (color online). (a) Phase of precessing spins after optical excitation with linearly polarized light at different incident polarization angles $\varphi$ near the sign reversal in Figs. 2(b) and 2(c). (b) Illustration of spins oriented in polar and transverse direction and respective TRFR curve as expected in the polar observation direction. According to the measured phase, $\theta_{F}$ will be decomposed into polar and transverse amplitudes plotted as a function of (c) incident linear polarization direction and (d) external magnetic field.

$\delta=-180^{\circ}$. This explains the sign reversal between points $A$ and $B$ in Fig. 2(c). In contrast, for a phase of $\delta=-90^{\circ}$ spin precession starts with $\theta_{F}=0^{\circ}$ at $\Delta t=0 \mathrm{~ns}$, which is illustrated in Fig. 3(b). This unambiguously demonstrates that spins will be oriented along the $x$ axis (transverse to both incident light direction and magnetic field direction) at the respective linear polarization angle. It is important to note that the excitation of transverse spins is unique to the optical orientation with linearly polarized light. It has not been observed for excitation with circularly polarized light. Knowing that we can excite both polar and transverse spins with linearly polarized light, we can now decompose the projections for any polarization angle along the polar and the transverse direction using $\theta_{p}=\theta_{0} \cos \delta$ and $\theta_{t}=\theta_{0} \sin \delta$, respectively. As shown in Fig. 3(c), polar and transverse spin signals change sign at different pump polarization angles. By carefully adjusting the polarization angle $\varphi$ we achieve a full two-dimensional control over the initial spin orientation, which cannot be realized by any other electrical or optical technique.

To further explore the origin of these unexpected findings, we plot the magnetic field dependence of both polar and transverse spin components in Fig. 3(d). Note that the amplitude is a measure of the number of electron spins initially oriented in the respective direction. The amplitude of the polar spins (black squares) is almost independent of magnetic field, while the amplitude of the transverse spins [gray (red) squares] depends linearly on the magnetic field and vanishes at $B=0 \mathrm{~T}$. This shows that transverse spins can only be excited at nonzero magnetic fields. We note that the transverse spin orientation switches sign from $+x$ to $-x$ direction for negative $B$ values. Such a linear $B$ dependence has previously been reported in photocurrent measurements where spins are optically generated by infrared absorption. The underlying intrinsic theory of the so-called magnetogyrotropic photogalvanic effect [13] relates the spin orientation directly to the underlying crystal structure. We can easily test whether the observed effect is related to the crystal axis by comparing the linear polarization dependence of $\theta_{0}$ for different sample orientations. In Fig. 4(a) we compare the dependence of the Faraday amplitudes $\theta_{p}$ and $\theta_{t}$ on $\varphi$ with the [1 110$]$ crystal axis either oriented parallel (left-hand panel) or under $26^{\circ}$ (right-hand panel) with respect to the external magnetic field direction [see also Fig. 1(a)] using polar plots. The amplitudes have been extracted from the TRFR data using the above analysis [14]. It is obvious that the observed symmetry is independent of the crystal orientation. The same behavior was also observed for other angles at $45^{\circ}$ and $90^{\circ}$ (not shown) suggesting that our effect is of extrinsic origin. This excludes various intrinsic effects as a source for the observed spin polarization, such as spin polarization induced by the Dresselhaus fields [15], intrinsic spin Hall effect [16], intrinsic double refraction and dichroism $[17,18]$, and inverse Faraday and Cotton-Mouton effect $[19,20]$.

We note that the observed spin polarization becomes largest for both polar and transverse spin components at an incident polarization direction of, e.g., $45^{\circ}$ [see Fig. 4(a)], while it vanishes for $s$ and $p$ polarized light at $\varphi=0^{\circ}$ and $90^{\circ}$, respectively. Away from normal incidence, it is well known that the linear polarization state is conserved only for $s$ and $p$ polarized light. For other polarization angles,
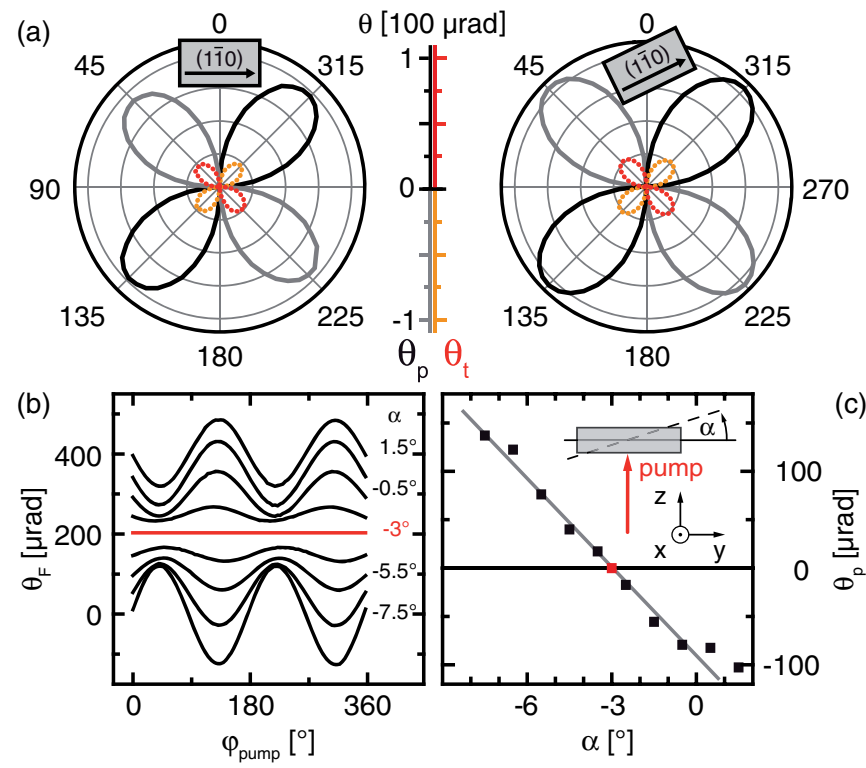

FIG. 4 (color online). (a) Polar diagrams of $\theta_{p}$ (black or gray) and $\theta_{t}$ (red or orange dotted lines) as a function of incident polarization direction for two different crystal orientations. (b) $\theta_{p}$ versus $\varphi$ at various angles of incidence $\alpha$. A vertical offset is added for clarity. (c) Linear dependence of $\theta_{p}$ plotted at $\varphi=40^{\circ}$. The sign reversal at $\alpha=-3^{\circ}$ shows a change in spin orientation of the polar spins near normal incidence. 
there will be an additional birefringence, which becomes largest for $\varphi=45^{\circ}$. At this angle, the light is elliptically polarized, which would result in spin orientation by the circular polarization component along the laser propagation direction. As the birefringence is negligible near normal incidence, it will be instructive to study the spin polarization away from normal incidence. We therefore rotated the sample about the $x$ axis by an angle $\alpha$ as defined in Fig. 1(a). In Figs. 4(b) and 4(c), we focus on the polar spin amplitude $\theta_{p}$. The data in Fig. 4(b) were taken at a fixed pump-probe delay of $\Delta t=1 \mathrm{~ns}$ with $B=0 \mathrm{~T}$ for different values of $\alpha$. As expected, the sign of $\theta_{p}$ oscillates as a function of $\varphi$. Surprisingly, there is a sign reversal of $\theta_{p}$ at $\alpha=-3^{\circ}$ [21], which is not seen for excitation with circularly polarized light (not shown), showing that the polar spin orientation can be switched into opposite orientations when rotating the sample from $\alpha<-3^{\circ}$ to $\alpha>-3^{\circ}$ [Fig. 4(c)]. This furthermore explains why we observe spins at normal incidence, i.e., at $\alpha=0^{\circ}$. Note that there is a remarkable increase of $\theta_{p}$ slightly away from $\alpha=-3^{\circ}$. At $\alpha \leq-7.5^{\circ}$ we find a value of $\theta_{p}$, which is $85 \%$ of the corresponding amplitude obtained for $\sigma^{+}$ excitation. Such a large number of spins cannot be explained by the above birefringence effect, which is estimated to be on the order of a few percent at $\alpha=-7.5^{\circ}$. We should emphasize that the birefringence in our optical setup is less than $1 \%$ and thus can also not explain the observed spin polarization.

Another possible source of spin polarization are local electric fields induced by the laser pulse. It was shown previously that static electric fields can indeed induce a spin polarization as demonstrated, e.g., in experiments on current-induced spin polarization [22] or extrinsic spin Hall effect [23]. In the latter case, spin currents of opposite polarization directions are generated perpendicular to the charge transport direction by skew scattering of electrons by the Coulomb potential of charged impurities. This scattering process does not depend on the crystal orientation. In our optical experiments, the laser pulse generates a nonequilibrium electron-hole population. As the mobility of holes is much lower than that of electrons, there will be a fast buildup of local electric fields, which can result in photocurrents on a picosecond time scale [24,25]. It is likely that these photocurrents are relevant in our experiments. In fact, optically triggered extrinsic spin Hall currents have been predicted in quantum well structures [26]. We furthermore note that in III-V quantum wells coherent spins could recently be excited in large magnetic fields after resonant light-hole excitation using arbitrarily polarized light $[27,28]$.

In conclusion, we have demonstrated that optical orientation of electron spins by linearly polarized light is very effective. From the phase during spin precession we can unambiguously prove that spins can be generated in both polar and transverse directions. This is in contrast to optical orientation by circularly polarized light for which spins are aligned in polar directions only. Surprisingly, the spin orientation is independent of the in-plane crystallographic directions of the sample, pointing to an extrinsic origin of the spin polarization. The full two-dimensional control over the initial spin direction offers the opportunity to study anisotropies in spin relaxation. This might give decisive clues about the dominating spin generation and relaxation mechanisms.

This work was supported by DFG through FOR 912.

*beschoten@physik.rwth-aachen.de

[1] M. I. D'yakonov and V.I. Perel, in Optical Orientation, edited by F. Meier and B.P. Zakharchenya (Elsevier Science, Amsterdam, 1984).

[2] I. Žutić, J. Fabian, and S. Das Sarma, Rev. Mod. Phys. 76, 323 (2004).

[3] S. A. Crooker and D. L. Smith, Phys. Rev. Lett. 94, 236601 (2005).

[4] R. I. Dzhioev et al., Phys. Rev. B 66, 245204 (2002).

[5] J. M. Kikkawa and D. D. Awschalom, Phys. Rev. Lett. 80, 4313 (1998).

[6] J.M. Kikkawa and D. D. Awschalom, Nature (London) 397, 139 (1999).

[7] A. V. Kimel et al., Phys. Rev. B 63, 235201 (2001).

[8] A. Greilich et al., Science 313, 341 (2006).

[9] L. Meier et al., Nature Phys. 3, 650 (2007).

[10] L. Schreiber et al., Phys. Rev. B 75, 193304 (2007).

[11] S. A. Tarasenko, Phys. Rev. B 72, 113302 (2005).

[12] S. D. Ganichev et al., Nature Phys. 2, 609 (2006).

[13] V. V. Bel'kov and S. D. Ganichev, Semicond. Sci. Technol. 23, 114003 (2008).

[14] There is a slight amplitude difference for polarization rotations of $\Delta \varphi=180^{\circ}$, which are related the retardation plate and which has been subtracted for simplicity.

[15] G. Dresselhaus, Phys. Rev. 100, 580 (1955).

[16] J. Sinova et al., Phys. Rev. Lett. 92, 126603 (2004).

[17] A. R. Bungay, N. Kugler, and N. I. Zheludev, Phys. Lett. A 174, 335 (1993).

[18] A. R. Bungay et al., Chem. Phys. Lett. 217, 249 (1994).

[19] A. V. Kimel et al., Nature (London) 435, 655 (2005).

[20] A. M. Kalashnikova et al., Phys. Rev. Lett. 99, 167205 (2007).

[21] We observe the sign reversal in all samples. However, the angle of sign reversal depends on the particular wafer. This sample to sample variation might be due to wafer miscuts or to specific properties of epilayer crystallinity.

[22] Y. K. Kato et al., Phys. Rev. Lett. 93, 176601 (2004).

[23] Y. K. Kato et al., Science 306, 1910 (2004).

[24] M. C. Nuss, D. H. Auston, and F. Capasso, Phys. Rev. Lett. 58, 2355 (1987).

[25] S. Priyadarshi et al., Phys. Rev. Lett. 104, 217401 (2010).

[26] E. Ya. Sherman et al., Solid State Commun. 139, 439 (2006).

[27] H. Kosaka et al., Phys. Rev. Lett. 100, 096602 (2008).

[28] H. Kosaka et al., Nature (London) 457, 702 (2009). 